INRA Prod. Anim.

2012, 25 (2), 101-112

\title{
L'organisation du travail en exploitation d'élevage : analyse de 630 Bilans Travail réalisés dans huit filières animales
}

\author{
S. COURNUT $1,2,3,4$, S. CHAUVAT 5 \\ ${ }^{1}$ Clermont Université, VetAgro Sup, UMR Métafort, BP 35, F-63370 Lempdes, France \\ 2 INRA, UMR1273 Métafort, F-63122 Saint-Genès-Champanelle, France \\ 3 AgroParisTech, UMR Métafort, BP 90054, F-63172 Aubière, France \\ 4 Irstea, UMR Métafort, BP 50085, F-63172 Aubière, France \\ 5 Institut de l'Elevage, SupAgro, F-34060 Montpellier, France \\ Courriel : sylvie.cournut@vetagro-sup.fr
}

Le travail est au centre des questions de pérennité des exploitations d'élevage. Les éleveurs souhaitent améliorer leurs conditions de travail et sont demandeurs de références permettant d'évaluer leurs marges de progrès. Pour les aider à réfléchir sur l'organisation du travail, les conseillers doivent pouvoir appréhender au mieux la situation de chaque exploitation et disposer de références adaptées.

Le travail en élevage est en pleine mutation. Les conditions d'exercice de l'activité d'élevage sont marquées par l'augmentation de la taille des exploitations, conjuguée à une réduction de la main-d'œuvre familiale et au développement du travail à l'extérieur des femmes (Johnsen 2004, Rattin 2006, Agreste 2011). La société et les marchés exercent des pressions croissantes sur les processus de production et modifient le rapport au travail d'une profession où l'astreinte aux animaux pèse sur le renouvellement de la population d'éleveurs (Ferris et al 2006, Madelrieux et Dedieu 2008). S'intéresser au travail des éleveurs et à la vivabilité des exploitations devient primordial (Gleeson et al 2008).

Pour appréhender le travail en exploitation d'élevage, les dimensions questionnées (productivité, durée, pénibilité, représentations, attentes...) et les cadres disciplinaires mobilisés (agronomie, sociologie, ergonomie, économie...) sont nombreux (Dedieu et al 2006). Cette étude s'appuie sur une représentation du travail en exploitation d'élevage formalisée par des zootechniciens, qui évalue l'organisation du travail par la quantification des temps de travaux sur une campagne de production: la méthode Bilan Travail (Dedieu et al 2000). Cette démarche a été largement utilisée en France (Seegers et al 2003, CaramelleHoltz et al 2004, Murat 2009), en Belgique (Turlot et al 2011) et dans des pays du sud (Dieguez et al 2010, Hostiou et al 2010, Correa et al 2011). Des références de temps de travaux par système de production sont construites et utilisées pour du conseil individuel ou collectif. L'analyse de la diversité des situations décrypte les principaux facteurs explicatifs des écarts de temps de travaux et des marges de manœuvre en temps des éleveurs (Cournut et Jordan 2008).

Le plus souvent, les comparaisons sont faites intra-filière car il est plus facile d'établir des repères de temps de travail lorsque les dimensions, les conduites et les équipements des systèmes d'élevage sont proches. Pourtant, si l'on dépasse ce cloisonnement, d'autres connaissances peuvent être produites. L'analyse comparée de l'organisation du travail dans des exploitations familiales, conduite en France, en Amérique latine et au Vietnam par Cournut et al (2010) le montre bien. En utilisant des techniques adaptées pour rendre possible l'analyse conjointe de situations extrêmes (e.g. des Surfaces Agricoles Utiles (SAU) de 7 ha au Vietnam vs 1700 ha dans la Pampa argentine), cette étude a montré que dans tous les pays, les éleveurs mobilisaient les mêmes leviers pour organiser le travail sur leur exploitation (conduite technique, équipements, combinaison et dimension des activités, et configuration de la maind'œuvre) et que la manière dont ces leviers étaient agencés témoignait de cohérences particulières dont on pou- vait retrouver l'expression dans tous les pays.

La mise en perspectives de données Bilan Travail issues de plusieurs filières, fait à la fois émerger les spécificités de chacune d'elles et révèle aussi certaines dimensions transversales aux filières comme les leviers d'organisation (recours au salariat par exemple) ou les logiques d'organisation (Cournut et Chauvat 2010b) qui correspondent aux cohérences particulières d'organisation s'exprimant dans la combinaison des différents leviers d'organisation du travail. Dans le cadre du Réseau Mixte Technologique (RMT) Travail en Elevage (Kling-Eveillard et al 2010), des Bilans Travail ont été réalisés dans 630 exploitations pour actualiser les référentiels de temps de travaux des filières herbivores (bovin laitier et à viande, ovin laitier et à viande, caprin laitier et fromager) et les étendre aux filières monogastriques (porc et volaille). Dans cette étude, nous avons utilisé ces données pour identifier $i$ ) le poids des orientations de production des exploitations, les spécificités ou points communs entre filières, ii) l'existence de logiques d'organisation particulières et leurs liens avec les filières et les collectifs qui gèrent l'exploitation.

Pour ce faire, nous nous sommes appuyés sur le cadre d'analyse de la méthode Bilan Travail et avons procédé en deux étapes correspondant aux deux points précédents. 


\section{1 / Un dispositif basé sur des enquêtes en exploitation}

Les exploitations enquêtées font partie des réseaux d'élevage pour le conseil et la prospective (Delaveau et al 1999) auxquelles ont été ajoutées 50 exploitations porcines et avicoles des régions Bretagne et Pays de la Loire. Les exploitations ovines laitières sont issues du bassin de Roquefort et des PyrénéesAtlantiques. En filière caprine, des livreurs de lait et des fromagers fermiers ont été enquêtés principalement dans le Centre Ouest et le Sud Est. La distinction entre fromager et laitier s'avère nécessaire car le cumul des activités de fabrication et de vente de fromages pèse fortement sur l'organisation du travail des élevages fromagers. Elle n'a pas été réalisée en bovin laitier car seulement trois élevages de l'échantillon transformaient leur lait en fromage, cette transformation ne concernant jamais la totalité du lait produit. Les exploitations des filières bovines et ovines à viande sont réparties sur l'ensemble du territoire français. Pour la constitution de cet échantillon, ni la représentativité, ni l'équilibre des exploitations entre filières n'ont été recherchés. Les enquêtes ont été réalisées par les techniciens, très majoritairement des chambres d'Agriculture, en 2008 et 2009.

A l'issue de la vérification des données, 9 exploitations enquêtées n'ont pas été conservées ; notre échantillon de travail comprend ainsi 631 exploitations (tableau 1).

\section{1 / Une démarche qui s'appuie sur la méthode Bilan Travail}

Nous appuyant sur la méthode du Bilan Travail (encadré 1), nous avons retenu trois volets d'analyse de l'organisation du travail :

- les temps de travaux quantifiés selon leur nature en heures (Travail d'Astreinte : TA) ou jours (Travail de Saison :TS),

\section{Encadré 1. La méthode Bilan Travail.}

Cette méthode, mise au point par l'INRA et l'Institut de l'Elevage, est basée sur un entretien avec les éleveurs qui vise à reconstituer l'emploi du temps des travailleurs à l'échelle de la campagne agricole. Elle quantifie le travail lié à la conduite des troupeaux et des surfaces, en distinguant les types de travaux et les catégories de main-d'œuvre.

Deux types de travaux sont distingués selon leur rythme de réalisation

- Le Travail d'Astreinte (TA). II s'effectue quotidiennement, il est difficile à concentrer et surtout peu différable. Pour l'élevage, il correspond aux soins journaliers apportés aux animaux (surveillance, alimentation, assistance aux mises bas...). Il est quantifié en heures par jour :

- Le Travail de Saison (TS). II réunit les tâches plus faciles à différer ou à concentrer. II porte sur i) les grandes cultures (TSCult), ii) la surface fourragère (TSSF), iii) les troupeaux (TSTH pour les herbivores et TSnonpât pour les monogastriques) ainsi que iv) l'entretien du territoire (TSET) comme l'entretien des haies et des clôtures. II est quantifié en jours par quinzaine.

Moins que la nature des tâches, ce sont le rythme d'accomplissement, l'échelle de mesure de temps (l'heure ou la journée) et la possibilité de différer ou non le travail qui sont les clés de ventilation. La tonte, par exemple, est comptabilisée dans le travail de saison pour un chantier de 2 jours à 5 personnes et dans le travail d'astreinte lorsque l'éleveur tond ses brebis au moment de l'agnelage pendant un mois. De la même façon, le nettoyage des bâtiments en volaille est comptabilisé dans le travail de saison car il correspond à des chantiers de une à plusieurs journées entre les bandes d'animaux, alors que le lavage des locaux en élevage porcin est comptabilisé en travail d'astreinte car il est non différable puisque soumis au mouvement des différents groupes d'animaux (maternité, sevrage, post-sevrage, engraissement) et comptabilisé en heures.

On distingue deux catégories de main-d'œuvre :

- La Cellule de Base (CB) est constituée des travailleurs permanents pour lesquels l'activité agricole est prépondérante en temps et en revenu et qui organisent le travail de l'exploitation (l'agriculteur, le couple d'exploitants, les associés d'un groupement agricole d'exploitation en commun...).

- La main-d'œuvre hors cellule de base regroupe les bénévoles (retraités, personnes donnant des coups de main), l'entraide, le salariat et l'intervention d'entreprises.

L'analyse permet la caractérisation et la quantification des travaux d'astreinte et de saison au niveau de l'exploitation et pour chaque catégorie de main-d'œuvre. Elle permet de construire des indicateurs d'efficience (heures ou jours par unité de production ou produite). Elle évalue pour la cellule de base, le "Temps Disponible Calculé» (TDC), qui est un indicateur de la marge de manœuvre en temps. II correspond au temps qui reste à la cellule de base pour les tâches non comptabilisées (entretien matériel, bâtiment, comptabilité, tâches administratives...) et les activités privées, une fois réalisée sa part de travail d'astreinte et de travail de saison. II est calculé en heures par an.

- la répartition de ce travail entre les différents travailleurs,

- le travail réalisé par personne de la Cellule de Base (pCB) et la marge de manœuvre en temps (Temps Disponible Calculé : TDC) dont elle dispose.

Dans la première étape de notre étude, nous analysons l'organisation du travail selon chacun des volets indiqués cidessus, en décrivant la variabilité et l'influence de la filière et du nombre de personnes de la cellule de base (Cournut et Chauvat 2010a). Dans la deuxième étape, l'analyse de l'organisation du travail porte conjointement sur les trois volets en identifiant des cohérences particulières que nous interprétons comme des logiques d'organisation.

\section{2 / Un traitement de données en deux étapes}

Les exploitations sont décrites par des variables de structure (tableau 1) et de caractérisation de l'organisation du travail. Nous avons construit une variable «mixité» en considérant comme mixte toute exploitation présentant en plus de sa production principale, des grandes cultures (plus de 40 ha), un atelier hors sol, des cultures spéciales ou un autre atelier herbivore.

Le traitement des données a été réalisé en suivant les deux étapes de notre analyse, à l'aide du logiciel Spad 7.0.

a) Première étape: analyse de la variabilité de l'organisation du travail

Nous avons décrit la distribution de chaque variable et analysé les différences entre filières, selon le nombre de $\mathrm{pCB}$, et entre élevage mixte et spécialisé, à l'aide d'analyses de variance mono facteur et de tests Newman-Keuls de comparaison multiples de moyennes. Nous avons retenu deux niveaux de facteur pour le nombre de $\mathrm{pCB}(\mathrm{pCB}=1$ et $\mathrm{pCB}=2$ et plus) car les premières analyses ont montré qu'il n'y avait pas de différences entre les niveaux $\mathrm{pCB}=2$ et $\mathrm{pCB}=3$ et plus.

\section{b) Deuxième étape : identification de} logiques d'organisation

Nous avons réalisé une Analyse en Composantes Principales (ACP) suivie d'une Classification Ascendante Hiérarchique $(\mathrm{CAH})$ (Escofier et Pagès 1998) sur l'ensemble de l'échantillon. Chaque exploitation était décrite par 10 variables (tableau 2) relatives aux dimensions, aux temps de travaux et à la répartition du travail entre la $\mathrm{CB}$ et la main-d'œuvre hors $\mathrm{CB}$ et au temps disponible calculé. A ces variables actives, nous avons adjoint des variables illustratives quantitatives (UGB et 
Tableau 1. Caractéristiques structurelles des exploitations selon les filières.

\begin{tabular}{|c|c|c|c|c|c|c|c|c|c|}
\hline Filières & $\begin{array}{c}\text { Bovin } \\
\text { lait }\end{array}$ & $\begin{array}{l}\text { Bovin } \\
\text { viande }\end{array}$ & $\begin{array}{c}\text { Caprin } \\
\text { fromager }\end{array}$ & $\begin{array}{c}\text { Caprin } \\
\text { lait }\end{array}$ & $\begin{array}{l}\text { Ovin } \\
\text { lait }\end{array}$ & $\begin{array}{l}\text { Ovin } \\
\text { viande }\end{array}$ & Porc & Volaille & Ensemble \\
\hline Nombre exploitations & 185 & 167 & 17 & 32 & 62 & 126 & 22 & 20 & 631 \\
\hline$\%$ mixtes $^{1}$ & 45 & 38 & 24 & 59 & 39 & 56 & 73 & 65 & 47 \\
\hline $\begin{array}{l}\% \text { avec atelier grandes } \\
\text { cultures }^{2}\end{array}$ & 26 & 30 & 6 & 28 & 5 & 17 & 64 & 25 & 24 \\
\hline $\begin{array}{l}\% \text { avec } 2 \text { ateliers } \\
\text { herbivores }\end{array}$ & 16 & 4 & 6 & 34 & 32 & 33 & & & 17 \\
\hline$\%$ de $p C B=1$ & 28 & 51 & 29 & 38 & 31 & 57 & 59 & 75 & 43 \\
\hline SAU (moyenne - ha) & 101 & 139 & 22 & 75 & 72 & 97 & 67 & 45 & 101 \\
\hline SFP (moyenne - ha) & 70 & 108 & 12 & 32 & 60 & 76 & 4 & 17 & 73 \\
\hline $\begin{array}{l}\text { Grandes cultures } \\
\text { (moyenne - ha) }\end{array}$ & 32 & 34 & 7 & 39 & 14 & 27 & 55 & 29 & 30 \\
\hline UGB (moyenne) & 96 & 134 & 19 & 56 & 72 & 96 & & & 100 \\
\hline $\begin{array}{l}\text { Unités productrices } \\
\text { (moyenne) }\end{array}$ & $\begin{array}{c}55 \\
\text { vaches }\end{array}$ & $\begin{array}{c}80 \\
\text { vaches }\end{array}$ & $\begin{array}{c}86 \\
\text { chèvres }\end{array}$ & $\begin{array}{c}235 \\
\text { chères }\end{array}$ & $\begin{array}{c}398 \\
\text { brebis }\end{array}$ & $\begin{array}{c}486 \\
\text { brebis }\end{array}$ & $\begin{array}{l}160 \\
\text { truies }\end{array}$ & & \\
\hline SAU/pCB (moyenne - ha) & 55 & 93 & 11 & 42 & 39 & 71 & 50 & 35 & 64 \\
\hline UGB/pCB (moyenne) & 53 & 91 & 11 & 36 & 41 & 68 & & & 64 \\
\hline
\end{tabular}

1 Au moins deux ateliers animaux ou au moins un atelier animal et des cultures.

2 Atelier cultures : surface en grandes cultures supérieure à 40 ha.

pCB : nombre de personnes de la Cellule de Base ; SAU : Surface Agricole Utile ; SFP : Surface Fourragère Principale ; UGB : Unité Gros Bétail.

$\mathrm{UGB} / \mathrm{pCB}^{1}$; surfaces de grandes cultures; \% TA réalisé par le bénévolat, le salariat et la CB; efficience : TA herbivore/UGB 2 et TS SFP/ha de SFP) et qualitatives (filières, $\mathrm{pCB}$, mixité et région). La caractérisation des groupes d'exploitations obtenus suite à la classification, a été menée à l'aide d'analyses de variance et comparaisons multiples de moyenne (NewmanKeuls) pour les variables quantitatives, et à l'aide de tests du Chi2 pour les variables qualitatives.

Tableau 2. Liste des variables de l'analyse en composantes principales.

\begin{tabular}{|l|l|}
\hline Critère & \multicolumn{1}{|c|}{ Description } \\
\hline Dimension & Surface Agricole Utile (ha) \\
\hline SAU & Surface Agricole Utile par personne de la Cellule de Base (ha) \\
\hline SAU/pCB & Travail d'Astreinte annuel (h) \\
\hline Temps de travaux, répartition du travail \\
\hline TA & Travail d'Astreinte annuel fait par la Cellule de Base (h) \\
\hline TACB & $\begin{array}{l}\text { Travail d'Astreinte annuel fait par la Cellule de Base par personne } \\
\text { de la Cellule de Base (h) }\end{array}$ \\
\hline TACB/pCB & Travail de Saison annuel (j) \\
\hline TS & Travail de Saison annuel fait par la Cellule de Base (j) \\
\hline TSCB & $\begin{array}{l}\text { Travail de Saison annuel fait par la Cellule de Base par personne } \\
\text { de la Cellule de Base (j) }\end{array}$ \\
\hline TSCB/pCB & Temps Disponible Calculé (h) \\
\hline Marge de mancuvre & Temps Disponible Calcule par personne de la Cellule de Base $(h)$ \\
\hline TDC &
\end{tabular}

pCB : nombre de personnes de la Cellule de Base.

\section{2 / Principaux déterminants de l'organisation du travail}

\section{1 / Un échantillon où l'imbri- cation entre filière et taille de la cellule de base est forte}

Les principales caractéristiques de l'échantillon sont présentées dans le tableau 1. Les plus grandes structures s'observent dans les élevages bovins et ovins à viande, les plus petites dans les élevages caprins fromagers. Les surfa- ces en grandes cultures sont plus importantes dans les élevages porcins.

Au total, 294 exploitations soit 47\% de notre échantillon ne sont pas spécialisées. Pour la moitié d'entre elles $(\mathrm{n}=$ 153), l'élevage est associé à un atelier de grandes cultures. Ensuite, la combinaison la plus fréquente concerne les doubles ateliers herbivores $(\mathrm{n}=110)$, le deuxième atelier étant presque toujours bovin allaitant. Les élevages mixtes se rencontrent dans toutes les filières mais sont moins fréquents en élevages caprins fromagers qui cumulent activités de fabrication et de vente de fromages. La présence d'un atelier de grandes cultures concerne les deux tiers des élevages porcins alors qu'elle est rare dans les élevages ovins laitiers et caprins fromagers. La mixité est plus fréquente dans les élevages gérés par des couples ou des associés (51\% des exploitations avec $\mathrm{pCB}>=2$ ) que dans ceux conduits par une seule personne (4\% quand $\mathrm{pCB}$ $=1$ ).

Les exploitations gérées par une ou deux personnes ( $\mathrm{pCB}=1$ ou 2 ) représentent chacune $43 \%$ de l'échantillon. Dans les filières avec production laitière (bovin, ovin, caprin), les exploitations conduites par une seule personne sont minoritaires (moins d'un tiers) alors qu'elles sont majoritaires dans les autres filières (près des trois quarts des exploitations de la filière volaille). L'imbrication entre la filière et la taille

\footnotetext{
${ }^{1}$ Les variables illustratives UGB et UGB/pCB caractérisent uniquement les élevages de ruminants.

${ }^{2} \mathrm{La}$ variable illustrative TA herbivore/UGB caractérise uniquement l'efficience des élevages de ruminants.
} 
Figure 1. Répartition par filière (en \%) des cellules de base selon leur taille. pCB : nombre de personnes de la Cellule de Base.

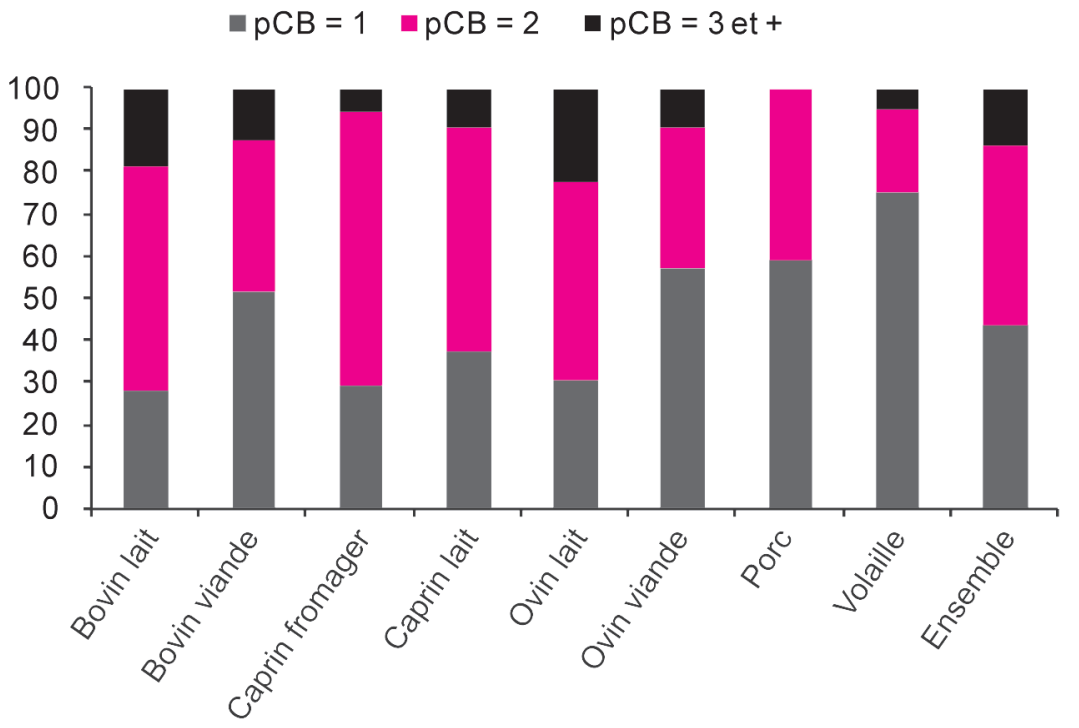

Tableau 3. Travail d'astreinte annuel moyen par filière selon la taille de la cellule de base.

\begin{tabular}{|l|c|c|c|}
\hline \multicolumn{1}{|c|}{ Filière } & $\begin{array}{c}\text { Travail d'astreinte } \\
\text { pour pCB =1 }\end{array}$ & $\begin{array}{c}\text { Travail d'astreinte } \\
\text { pour pCB = 2 et + }+^{(1)}\end{array}$ & $\begin{array}{c}\text { Travail d'astreinte } \\
\text { Ensemble }\end{array}$ \\
\hline Bovin lait & $2150(598)$ & $3320(1352)$ & $2990^{(i 2)}(1300)$ \\
\hline Bovin viande & $1660(702)$ & $2590(1340)$ & $2110^{\theta}(1160)$ \\
\hline Caprin fromager & $3820(1630)$ & $5600(2120)$ & $5080^{\mathrm{a}}(2110)$ \\
\hline Caprin lait & $3250(1420)$ & $3730(1380)$ & $3550^{\mathrm{bc}}(1390)$ \\
\hline Ovin lait & $3350(1540)$ & $3690(930)$ & $3590^{\mathrm{b}}(1150)$ \\
\hline Ovin viande & $2070(910)$ & $3160(1460)$ & $2540^{\mathrm{d}}(1290)$ \\
\hline Porc & $3290(1172)$ & $3830(1130)$ & $3510^{\mathrm{bc}}(1160)$ \\
\hline Volaille & $1880(1385)$ & $3170(2330)$ & $2200^{\mathrm{de}}(1700)$ \\
\hline Ensemble & $2180(1103)$ & $3280(1470)$ & $2800(1432)$ \\
\hline
\end{tabular}

(1) Moyenne (écart-type) en heures.

(2) Les lettres en exposant correspondent à la comparaison des moyennes entre filières : les moyennes avec une lettre commune en exposant ne sont pas significativement différentes $(P<0,05)$

pCB : nombre de personnes de la Cellule de Base.

Tableau 4. Travail de saison annuel moyen par filière selon la taille de la cellule de base.

\begin{tabular}{|l|c|c|c|}
\hline \multicolumn{1}{|c|}{ Filière } & $\begin{array}{c}\text { Travail de saison } \\
\text { pour } \mathrm{pCB}=\mathbf{1}^{(1)}\end{array}$ & $\begin{array}{c}\text { Travail de saison } \\
\text { pour pCB =2 et }+^{(1)}\end{array}$ & $\begin{array}{c}\text { Travail de saison } \\
\text { Ensem ble }\end{array}$ \\
\hline Bovin lait & $99(53)$ & $167(105)$ & $148^{\mathrm{b}(2)}(98)$ \\
\hline Bovin viande & $158(73)$ & $248(115)$ & $202^{\mathrm{a}}(106)$ \\
\hline Caprin fromager & $20(5)$ & $72(49)$ & $57^{\mathrm{d}}(47)$ \\
\hline Caprin lait & $71(39)$ & $164(77)$ & $129^{\mathrm{bc}}(79)$ \\
\hline Ovin lait & $114(48)$ & $175(85)$ & $156^{\mathrm{ab}}(80)$ \\
\hline Ovin viande & $135(63)$ & $200(85)$ & $163^{\text {ab }}(80)$ \\
\hline Porc & $76(70)$ & $113(47)$ & $91^{\mathrm{cd}}(63)$ \\
\hline Volaille & $130(52)$ & $216(93)$ & $151^{\mathrm{ab}}(73)$ \\
\hline Ensemble & $126(69)$ & $187(106)$ & $161(97)$ \\
\hline
\end{tabular}

(1) Moyenne (écart-type) en jours.

(2) Les lettres en exposant correspondent à la comparaison des moyennes entre filières :

les moyennes avec une lettre commune en exposant ne sont pas significativement différentes $(\mathrm{P}<0,05)$

pCB : nombre de personnes de la Cellule de Base. de la cellule de base (figure 1) est donc forte.

\section{2 / L'organisation du travail est marquée par les filières}

\section{a) Les temps de travaux}

Le travail d'astreinte. Le TA moyen par exploitation est de 2800 heures par an (tableau 3). Les disparités entre filières sur le volume annuel d'heures de travail d'astreinte $(\mathrm{P}<0,001)$ mais aussi entre les exploitations à une personne dans la $\mathrm{CB}$ et les autres $(\mathrm{P}<0,001)$ sont très marquées. A contrario, il n'y a pas de différence entre systèmes mixtes et spécialisés sur le niveau de TA de l'exploitation. Les exploitations caprines fromagères se distinguent nettement avec un travail d'astreinte pratiquement double de la moyenne observée sur l'échantillon. La charge de travail due à la traite explique en partie le classement des résultats selon les filières, les élevages de petits ruminants cumulant le handicap d'un nombre important d'animaux à traire. Parmi les valeurs de TA les plus élevées figurent celles des élevages porcins : ceci résulte de la comptabilisation dans le TA des tâches qui ne peuvent pas être différées, en raison de la succession de bandes d'animaux, et correspondant au nettoyage des bâtiments et à la manipulation des animaux (incluses dans les travaux de saison pour les herbivores et les volailles). Les exploitations de bovins à viande et de la filière volaille sont les moins chargées en TA.

Le travail de saison. Le TS moyen par exploitation est de 161 jours par an. À l'instar du travail d'astreinte, la filière et le nombre de pCB influent significativement sur le TS $(\mathrm{P}<0,001)$ (tableau 4). Les exploitations des filières les moins chargées en travail d'astreinte sont celles qui ont le plus de travail de saison. Dans les élevages allaitants, cela s'explique par l'importance des effectifs d'animaux (80 vaches allaitantes en moyenne vs 55 vaches laitières ; 486 brebis allaitantes vs 398 brebis laitières) et des manipulations (traitements sanitaires, pesées, tonte, tris) souvent plus fréquentes et plus lourdes à mettre en œuvre (pas d'opportunité de contention individuelle comme pour la traite).

La nature du TS est aussi bien différente selon les filières (figure 2). Dans les élevages de porcs, le TS consacré aux animaux (TSnonpât) est faible par rapport à la filière volaille ( 25 jours en moyenne) et correspond principalement aux départs à l'abattoir de porcs charcutiers ou à l'éventuelle fabrication d'aliment fermier. Les élevages de la filière 
Figure 2. Composition du travail de saison annuel selon la filière.

TSSF : Travail de Saison sur la Surface Fourragère ; TSCult : Travail de Saison sur les grandes Cultures ; TSET : Travail de Saison pour l'Entretien du Territoire ; TSTH : Travail de Saison sur le Troupeau Herbivore ; TSnonpât : Travail de Saison sur les monogastriques.

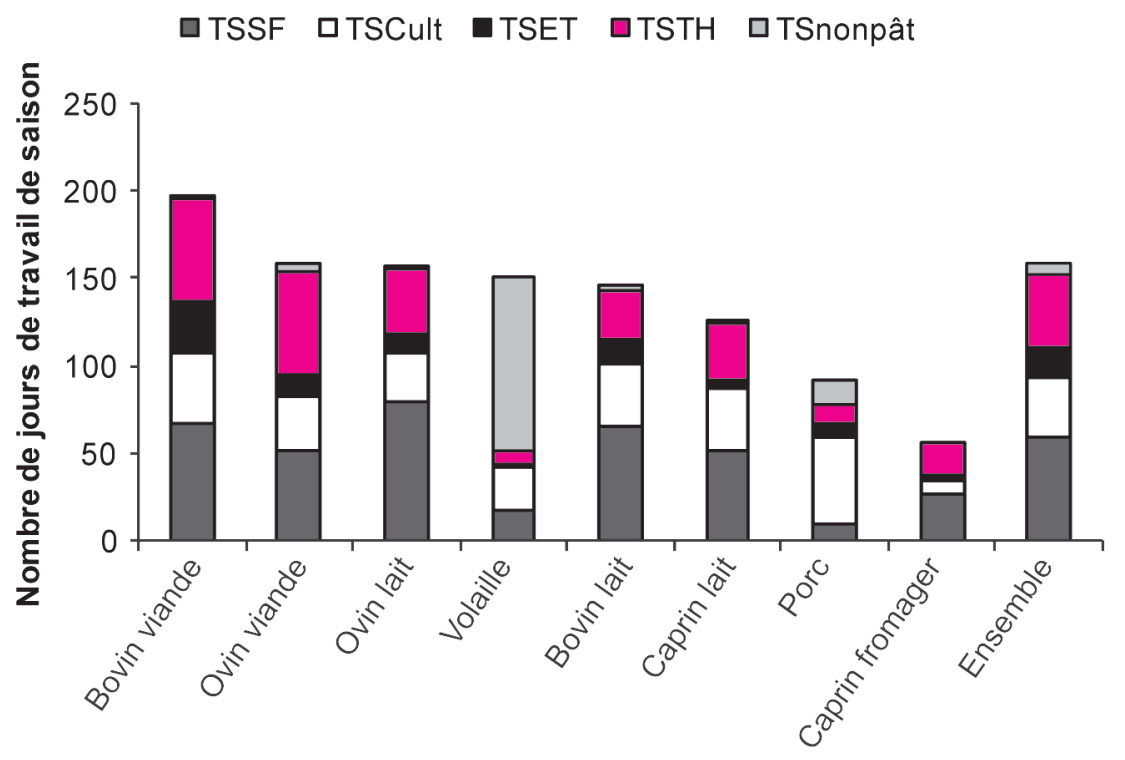

volaille consacrent en moyenne 98 jours de TS pour l'enlèvement des animaux et le nettoyage des bâtiments entre les bandes. Le Travail Sur les Surfaces Fourragères (TSSF) est important pour les élevages herbivores (65 à 79 jours), à l'exception des élevages caprins fromagers (36 jours) car les achats de fourrages y sont plus fréquents. Dans les exploitations ayant un atelier de grandes cultures, le TS atteint 210 jours (vs 144 jours par an pour les autres, $\mathrm{P}<0,001)$, du fait du travail sur les surfaces (TSCult de 86 jours contre 23 jours par an, $\mathrm{P}<0,001)$. Enfin, le travail d'entretien du territoire concerne toutes les fillières (en moyenne 21 jours) à 1'exception de la filière volaille. (respectivement de 77, 71 et $78 \%$ ) mais le travail hors $\mathrm{CB}$ correspond à des fonctionnements différents : le salariat est mobilisé dans les élevages caprins fromagers et porcins (réalisant respectivement 19 et $24 \%$ du TA), alors que le bénévolat est mobilisé dans les exploitations ovines laitières (pour 19\% du TA). Dans ce dernier cas, le taux est dû aux exploitations des Pyrénées-Atlantiques, pour lesquelles la part de bénévolat atteint $40 \%$. Reposant sur une participation parentale, il s'inscrit dans la logique de transmission patrimoniale des exploitations.

Pour le travail de saison (tableau 5), la délégation est plus importante puisque seulement $74 \%$ de ce travail est réalisé par la $\mathrm{CB}$, avec là encore des différences significatives selon la taille de celle-ci (65 vs 80\%, $\mathrm{P}<0,001$ ). Dans les deux filières monogastriques, la $\mathrm{CB}$ réalise seulement 64 et $53 \%$ du TS : ces exploitations recourent à l'entreprise pour les cultures dans les élevages porcins; pour l'enlèvement et le nettoyage des bâtiments entre bandes dans les élevages de volailles. La présence d'un atelier de grandes cultures est associée à plus de délégation à de la main-d'œuvre salariée $(10 \%$ du TA contre $4 \%$ pour l'ensemble des élevages et 11 contre $6 \%$ pour le TS).

Le bénévolat est présent dans $65 \%$ des exploitations où il intervient le plus souvent sur le TS à hauteur de 33 jours par an en moyenne. Quand il est mobilisé pour le travail d'astreinte, il réalise en moyenne 800 heures par an. La main-d'œuvre salariée intervient dans $20 \%$ des exploitations sur du travail d'astreinte où elle réalise en moyenne 860 heures par an, et dans 35\% des exploitations sur du TS à hauteur de 40 jours par an en moyenne. Le recours à l'entreprise pour le TS concerne la majorité des exploitations $(81 \%$ dont la

Tableau 5. Répartition moyenne du travail d'astreinte et du travail de saison selon le type de main-d'œuvre.

\begin{tabular}{|l|c|c|c|c|c|c|c|c|}
\hline \multicolumn{1}{|c|}{ Filière } & $\begin{array}{c}\% \text { TA } \\
\text { cellule } \\
\text { de base }\end{array}$ & $\begin{array}{c}\text { \% TA } \\
\text { bénévolat }\end{array}$ & $\begin{array}{c}\text { \% TA } \\
\text { salariat }\end{array}$ & $\begin{array}{c}\% \text { TS } \\
\text { cellule } \\
\text { de base }\end{array}$ & $\begin{array}{c}\text { \% TS } \\
\text { bénévolat }\end{array}$ & $\begin{array}{c}\% \text { TS } \\
\text { salariat }\end{array}$ & $\begin{array}{c}\% \text { TS } \\
\text { entreprise }\end{array}$ & $\begin{array}{c}\% \text { TS } \\
\text { entraide }\end{array}$ \\
\hline Bovin lait & 91 & 5 & 4 & 77 & 6 & 7 & 5 & 6 \\
\hline Bovin viande & 86 & 11 & 3 & 72 & 13 & 8 & 3 & 5 \\
\hline $\begin{array}{l}\text { Caprin } \\
\text { fromager }\end{array}$ & 77 & 4 & 19 & 73 & 4 & 15 & 8 & 0 \\
\hline Caprin lait & 83 & 7 & 11 & 80 & 7 & 6 & 5 & 3 \\
\hline Ovin lait & 78 & 19 & 3 & 71 & 11 & 7 & 6 & 5 \\
\hline Ovin viande & 85 & 11 & 4 & 76 & 11 & 6 & 4 & 2 \\
\hline Porc & 71 & 4 & 24 & 64 & 12 & 8 & 13 & 2 \\
\hline Volaille & 89 & 6 & 5 & 53 & 12 & 7 & 24 & 4 \\
\hline Ensemble & 86 & 9 & 5 & 74 & 10 & 7 & 5 & 4 \\
\hline
\end{tabular}


Tableau 6. Le travail d'astreinte de la cellule de base par pCB selon la filière et la taille de la cellule de base.

\begin{tabular}{|l|c|c|c|}
\hline \multicolumn{1}{|c|}{ Filière } & $\begin{array}{c}\text { Travail d'astreinte } \\
\text { de la CB/pCB } \\
\text { pour pCB = } \text { (1) }^{(1)}\end{array}$ & $\begin{array}{c}\text { Travail d'astreinte de } \\
\text { la CB/pCB } \\
\text { pour pCB = 2 et }+^{(1)}\end{array}$ & $\begin{array}{c}\text { Travail d'astreinte de } \\
\text { la CB/pCB } \\
\text { Ensem b/e(1) }\end{array}$ \\
\hline Bovin lait & $1730(450)$ & $1330(380)$ & $1440^{6 / 2)}(440)$ \\
\hline Bovin viande & $1230(390)$ & $1020(430)$ & $1130^{c}(420)$ \\
\hline Caprin fromager & $2390(490)$ & $2020(610)$ & $2130^{a}(590)$ \\
\hline Caprin lait & $2050(720)$ & $1530(460)$ & $1720^{6}(620)$ \\
\hline Ovin lait & $1940(500)$ & $1300(320)$ & $1500^{6}(480)$ \\
\hline Ovin viande & $1570(510)$ & $1220(470)$ & $1420^{b}(520)$ \\
\hline Porc & $1990(560)$ & $1360(280)$ & $1728^{b}(560)$ \\
\hline Volaille & $1530(780)$ & $1290(680)$ & $1470^{6}(740)$ \\
\hline Ensemble & $1570(770)$ & $1270(460)$ & $1400(530)$ \\
\hline
\end{tabular}

(1) Moyenne (écart-type) en heures.

(2) Les lettres en exposant correspondent à la comparaison des moyennes entre filières

les moyennes avec une lettre commune en exposant ne sont pas significativement différentes $(P<0,05)$.

CB : Cellule de Base ; $\mathrm{pCB}$ : nombre de personnes de la Cellule de Base.

totalité des élevages de volailles) mais pour seulement 8 jours en moyenne, valeurs très proches de l'entraide $(61 \%$ des exploitations pour 9 jours en moyenne).

\section{c) Le travail de la cellule de base}

Le travail d'astreinte restant à la charge de la cellule de base est en moyenne de 1400 heures par an et par personne (tableau 6). De 1570 heures quand la CB est unipersonnelle, il est significativement inférieur de 300 heures lorsque la $\mathrm{CB}$ est composée de plusieurs personnes (différence significative avec $\mathrm{P}<0,001)$. Les différences entre filières sont également significatives $(\mathrm{P}<0,001)$ et conduisent à distinguer les exploitations caprines fromagères avec 2130 heures et les exploitations qui produisent des bovins à viande avec 1125 heures, des autres élevages.

Le TS restant à la cellule de base s'élève à 74 jours par an et par personne (tableau 7) avec là encore des différences selon la taille de la CB (83 vs
67 jours, $P<0,001)$, les filières (95 jours en bovin à viande contre 22 en caprin fromager), et la présence ou non d'un atelier de grandes cultures (respectivement 89 jours $v s 69$ jours).

$\mathrm{Au}$ final, la marge de manœuvre en temps (TDC/pCB), qui est en moyenne de 1000 heures par an et pCB (tableau 8), est plus faible pour les éleveurs seuls $(\mathrm{pCB}=1)$ que pour les autres ( 850 heures vs 1100 heures, $\mathrm{P}<0,001)$. L'amplitude des variations est considérable, de 0 à 2000 heures par an. Les élevages caprins, lourdement chargés en travail d'astreinte, ont des disponibilités très faibles (moins de 700 heures par an) alors que les élevages bovins allaitants en ont le plus 1070 heures par an). Mais les écarts sont moins élevés qu'entre tailles de CB et la variabilité au sein d'une même filière est importante. Les situations les plus défavorables (TDC/pCB inférieur à 800 heures par an) qui représentent $30 \%$ de l'échantillon, sont plus fréquentes d'une part, dans les exploitations gérées par un éleveur seul, lesquelles concernent près de la moitié des élevages et d'autre part, en caprin fromager où ce taux atteint les $71 \%$. A contrario, ces situations sont les plus rares pour les élevages ovins laitiers (19\%).

En complément de l'évaluation de la marge de manœuvre en temps, l'importance du bénévolat est considérée comme une source de fragilité de l'organisation. Les exploitations pour lesquelles le temps d'astreinte réalisé par la main-d'œuvre bénévole égale ou dépasse le temps disponible global de la cellule de base représentent en moyenne $10 \%$ de notre échantillon, avec des proportions allant de $5 \%$ en bovin laitier et en volailles, jusqu'à $18 \%$ en caprin fromager et en porcin.

\section{3 / Des logiques d'organisation différentes selon les collectifs qui les mettent en place}

Dans cette étape, nous analysons la variabilité de l'organisation du travail et

Tableau 7. Le travail de saison de la cellule de base par personne dans la cellule de base selon la filière et la taille de la cellule de base.

\begin{tabular}{|l|c|c|c|}
\hline \multicolumn{1}{|c|}{ Filière } & $\begin{array}{c}\text { Travail de saison } \\
\text { de la } \mathrm{CB} / \mathrm{pCB} \\
\text { pour pCB }=\mathbf{1}^{(1)}\end{array}$ & $\begin{array}{c}\text { Travail de saison de } \\
\text { laCB/pCB } \\
\text { pour pCB }=2 \text { et }+^{(1)}\end{array}$ & $\begin{array}{c}\text { Travail de saison } \\
\text { de la CB/pCB } \\
\text { Ensem ble }{ }^{[1]}\end{array}$ \\
\hline Bovin lait & $67(30)$ & $60(34)$ & $62^{\mathrm{C}(2)}(33)$ \\
\hline Bovin viande & $102(43)$ & $88(33)$ & $95^{\mathrm{a}}(39)$ \\
\hline Caprin fromager & $10(6)$ & $27(21)$ & $22^{\mathrm{d}}(20)$ \\
\hline Caprin lait & $51(25)$ & $66(29)$ & $60^{\mathrm{c}}(28)$ \\
\hline Ovin lait & $72(31)$ & $58(27)$ & $62^{\mathrm{c}}(29)$ \\
\hline Ovin viande & $95(45)$ & $74(27)$ & $86^{\mathrm{b}}(40)$ \\
\hline Porc & $41(25)$ & $39(13)$ & $40^{\mathrm{cd}}(21)$ \\
\hline Volaille & $69(36)$ & $66(37)$ & $68^{\mathrm{c}}(36)$ \\
\hline Tous & $83(44)$ & $67(34)$ & $74(40)$ \\
\hline
\end{tabular}

(1) Moyenne (écart-type) en jours

(2) Les lettres en exposant correspondent à la comparaison des moyennes entre filières :

les moyennes avec une lettre commune en exposant ne sont pas significativement différentes $(P<0,05)$.

CB : Cellule de Base ; pCB : nombre de personnes de la Cellule de Base. 
Tableau 8. Le temps disponible calculé par personne de la cellule de base selon la filière et la taille de la cellule de base.

\begin{tabular}{|l|c|c|c|}
\hline \multicolumn{1}{|c|}{ Filière } & $\begin{array}{c}\text { TDC/pCB } \\
\text { pour } \mathrm{pCB}=1^{(1)}\end{array}$ & $\begin{array}{c}\text { TDC/pCB } \\
\text { pour } \mathrm{pCB}=2 \text { et }+^{(1)}\end{array}$ & $\begin{array}{c}\text { TDC/pCB } \\
\text { Ensem ble }\end{array}$ \\
\hline Bovin lait & $790(330)$ & $1120(340)$ & $1030^{\mathrm{b}(2)}(370)$ \\
\hline Bovin viande & $980(330)$ & $1170(360)$ & $1070^{\mathrm{b}}(360)$ \\
\hline Caprin fromager & $560(380)$ & $740(410)$ & $690^{\mathrm{a}}(400)$ \\
\hline Caprin lait & $740(400)$ & $970(270)$ & $880^{\mathrm{ab}}(340)$ \\
\hline Ovin lait & $720(330)$ & $1140(260)$ & $1010^{\mathrm{b}}(340)$ \\
\hline Ovin viande & $830(340)$ & $1130(340)$ & $950^{\mathrm{ab}}(370)$ \\
\hline Porc & $710(400)$ & $1180(220)$ & $900^{\mathrm{ab}}(400)$ \\
\hline Volaille & $920(360)$ & $1030(370)$ & $950^{\mathrm{ab}}(360)$ \\
\hline Ensemble & $850(350)$ & $1110(340)$ & $1000(370)$ \\
\hline
\end{tabular}

(1) Moyenne (écart-type) en heures.

(2) Les lettres en exposant correspondent à la comparaison des moyennes entre filières :

les moyennes avec une lettre commune en exposant ne sont pas significativement différentes $(P<0,05)$.

TDC : Temps Disponible Calculé ; CB : Cellule de Base ; $\mathrm{PCB}$ : nombre de personnes de la Cellule de Base.

identifions et caractérisons des logiques d'organisation.

a) Les facteurs de variation de l'organisation du travail

Les trois premiers axes de l'ACP permettent d'expliquer $85 \%$ de la variance totale. Le premier axe $(33 \%$ de la variance) est un axe de dimension de l'exploitation. Il exprime un gradient lié, à la surface en grandes cultures, à la taille du cheptel et de la SAU, et au travail de saison. Il traduit le lien étroit entre l'augmentation des dimensions et celle de la quantité de TS, lien qui n'est pas observé pour le TA. Celui-ci intervient dans la construction du deuxième axe (30\% de la variance) en opposition avec le TDC/pCB, ce qui confirme que la marge de manœuvre en temps est essentiellement déterminée par le TA. Enfin, le troisième axe $(22 \%$ de la variance), dont l'inertie est liée aux dimensions (nombre d'animaux et d'hectares) rapportées à la personne de la $\mathrm{CB}$, oppose ces critères à la taille de la CB. La dimension des unités de production (cheptel et surface) par pCB est d'autant plus faible que la taille de la cellule de base est importante.

En résumé, la dimension de l'exploitation est un facteur majeur de diversité des situations de travail étudiées. Elle est directement liée à l'importance du TS, et corrélée aux surfaces en grandes cultures. La marge de manœuvre en temps ne dépend pas des dimensions mais est fortement liée à l'importance du TA.

\section{b) Les logiques d'organisation du tra-} vail

La classification ascendante hiérarchique, réalisée sur les coordonnées des trois axes issues de l'ACP, a permis d'identifier quatre groupes d'exploitations que nous interprétons comme des logiques différentes d'organisation du travail (tableau 9).

Tableau 9. Principales caractéristiques des quatre groupes d'exploitations.

\begin{tabular}{|c|c|c|c|c|c|c|}
\hline $\begin{array}{c}\text { Variables } \\
\text { caractéristiques }^{(1)}\end{array}$ & $\begin{array}{c}\text { G1 } \\
\text { Partage }\end{array}$ & $\begin{array}{c}\text { G2 } \\
\text { Délégation }\end{array}$ & $\begin{array}{c}\text { G3 } \\
\text { Ajustement }\end{array}$ & $\begin{array}{l}\text { G4 faible } \\
\text { marge de } \\
\text { manœuvre }\end{array}$ & Effet $^{(2)}$ & Ensemble \\
\hline Nombre exploitations & 115 & 135 & 213 & 168 & & 631 \\
\hline SAU (ha) & $163^{\text {a(3) }}(76)$ & $137^{\circ}(64)$ & $84^{\circ}(39)$ & $51^{d}(27)$ & $\star \star \star \star *$ & $101(66)$ \\
\hline Grandes cultures (ha) & $54^{a}(52)$ & $55^{a}(52)$ & $27^{\circ}(30)$ & $17^{c}(18)$ & $\star \star \star$ & $36(42)$ \\
\hline UGB & $152^{a}(78)$ & $115^{\circ}(63)$ & $81^{c}(42)$ & $56^{0}(35)$ & $\star \star \star *$ & $94(63)$ \\
\hline SAU/pCB (ha) & $65^{b}(30)$ & $117^{a}(50)$ & $46^{c}(21)$ & $43^{c}(25)$ & $\star \star \star *$ & $64(43)$ \\
\hline UGB/pCB & $60^{\circ}(33)$ & $101^{a}(56)$ & $46^{\circ}(26)$ & $47^{\circ}(32)$ & $\star * \star *$ & $60(43)$ \\
\hline TACB/pCB (h) & $1460^{\circ}(400)$ & $1190^{\circ}(390)$ & $1050(300)$ & $1980^{\circ}(430)$ & *** & $1400(530)$ \\
\hline TSCB/pCB (j) & $93^{\circ}(33)$ & $114^{a}(40)$ & $50^{\circ}(20)$ & $60^{\circ}(30)$ & $\star * \star *$ & $74(40)$ \\
\hline$\%$ TA CB & $89^{a}(15)$ & $80^{6}(22)$ & $89^{a}(17)$ & $84^{\circ}(20)$ & *** & $86(19)$ \\
\hline \% TA bénévolat & $8^{\mathrm{ab}}(13)$ & $12^{a}(17)$ & $7^{b}(14)$ & $10^{a b}(17)$ & * & $9(16)$ \\
\hline \% TA salariat & $3^{b}(9)$ & $8^{a}(16)$ & $4^{b}(11)$ & $6^{\mathrm{ab}}(14)$ & ${ }^{*}$ & $5(13)$ \\
\hline$\%$ TSCB & $81^{a}(16)$ & $67^{d}(19)$ & $76^{6}(17)$ & $71^{c}(19)$ & $* * *$ & $74(19)$ \\
\hline \% TS bénévolat & $8^{6}(11)$ & $12^{a}(16)$ & $8^{6}(13)$ & $11^{a b}(15)$ & ${ }^{*}$ & $10(14)$ \\
\hline \% TS salariat & $6^{6}(13)$ & $13^{a}(18)$ & $6^{b}(13)$ & $5^{b}(12)$ & *** & $7(14)$ \\
\hline TDC/pCB (h) & $880^{\circ}(270)$ & $950^{\circ}(310)$ & $1350^{a}(210)$ & $690^{d}(260)$ & $\star * \star$ & $1000(370)$ \\
\hline TS SFP/ha de SFP (j) & $1,1^{8}(0,7)$ & $0,8^{\circ}(0,9)$ & $0,9^{0}(0,6)$ & $1,3^{a}(1,0)$ & $\star \star \star \star$ & $1,0(0,8)$ \\
\hline TA herbivore/UGB (h) & $37^{\circ}(29)$ & $19^{d}(13)$ & $32^{c}(24)$ & $76^{a}(92)$ & $* * *$ & $41(54)$ \\
\hline
\end{tabular}

(1) Moyenne (écart-type).

(2) Signification statistique de l'analyse de variance : ${ }^{*} \mathrm{P}<0,05 ;{ }^{* *} \mathrm{P}<0,01 ;{ }^{* *} \mathrm{P}<0,001$

(3) Les lettres en exposant correspondent à la comparaison des moyennes entre groupes d'exploitations : les moyennes avec une lettre commune en exposant ne sont pas significativement différentes $(P<0,05)$.

CB : Cellule de Base ; pCB : nombre de Personnes de la Cellule de Base ; SAU : Surface Agricole Utile ; UGB : Unité Gros Bétail ;

TA : Travail d'Astreinte; TS : Travail de Saison ; TDC : Temps Disponible Calculé. 
La logique "partage» des grandes structures familiales (G1, $\mathrm{n}=115$ soit $18 \%$ de l'échantillon).

Les exploitations de ce groupe sont de grande taille (163 ha de SAU pour 152 UGB en moyenne), gérées le plus souvent par trois travailleurs (GAEC dont une majorité de successoraux). Ces élevages associent un atelier d'élevage de ruminants à un atelier de grandes cultures ou un autre atelier d'élevage (62\% d'élevages mixtes). Leur organisation est caractérisée par un recours relativement faible à de la main-d'œuvre hors cellule de base (seulement $11 \%$ du TA et $19 \%$ du TS est délégué); le travail est partagé entre personnes de la $\mathrm{CB}$. Bien que le bénévolat soit plus fréquent que dans les autres groupes (74\% des exploitations sont concernées), leur part de travail est faible (8\%). Les travaux de saison et d'astreinte sont importants et, malgré la taille du collectif, le travail de saison à la charge de chaque personne de la CB demeure élevé (93 jours par an). L'efficience du TA pour les herbivores (TAherb/UGB) et du TS sur la surface fourragère (TSSF/ha SFP) est moyenne et la marge de manœuvre (880 heures de TDC par an et $\mathrm{pCB}$ ) assez faible en regard de la taille de la cellule de base.

La logique «délégation» des grandes structures gérées par des éleveurs seuls (G2, $\mathrm{n}=135$ soit $21 \%$ de l'échantillon).

Ce groupe est composé d'exploitations de grandes dimensions gérées par une seule personne, pour laquelle la charge en surfaces et animaux s'avère la plus élevée de l'échantillon : 117 ha par pCB et 101 UGB par pCB. L'élevage est allaitant, bovin ou ovin. Comme dans le groupe précédent, les élevages mixtes

Figure 3. Répartition de la taille de la cellule de base (en \%) selon les logiques d'organisation du travail.

pCB : nombre de personnes de la Cellule de Base.

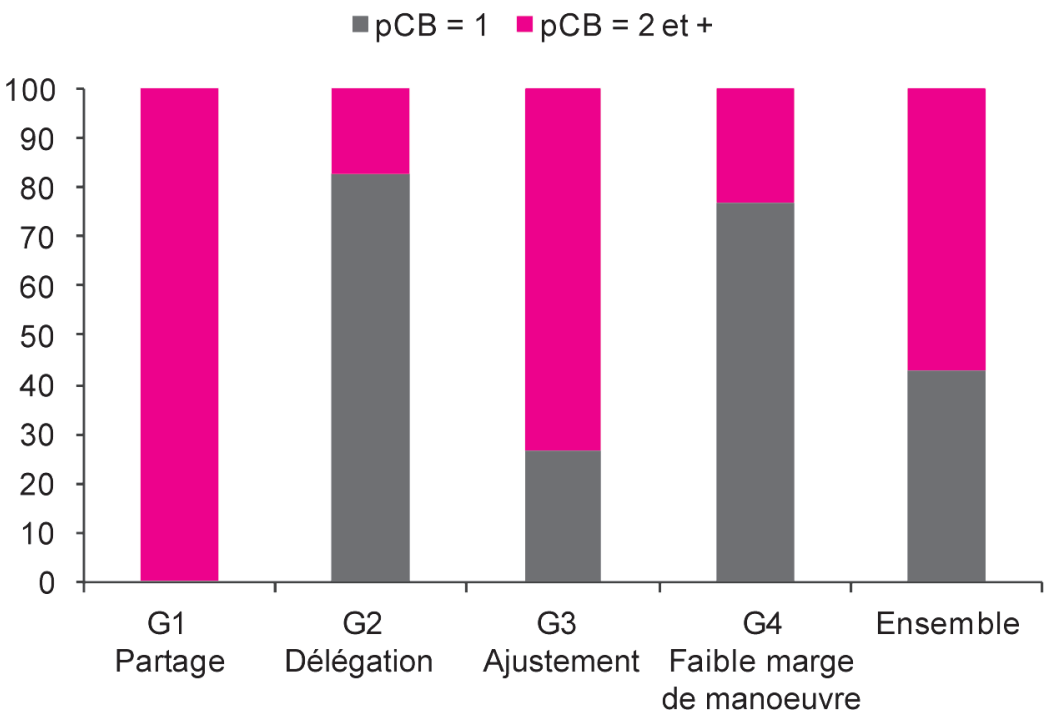

Le TS est le plus faible (50 jours par an et $\mathrm{pCB})$. La marge de manœuvre en temps est confortable (1350 heures par an et $\mathrm{pCB}$ ).

La logique «faible marge de manouvre» des petites structures gérées par des éleveurs seuls (G4, $\mathrm{n}=168$ soit $27 \%$ de l'échantillon).

Le groupe G4 regroupe des exploitations de petite taille ( 51 ha de SAU pour 56 UGB en moyenne) spécialisées et gérées par une seule personne dans les deux tiers des cas. L'organisation du travail est caractérisée par la charge élevée de travail d'astreinte de chaque personne de la CB (1980 heures par an) et le peu de travail de saison (60 jours par an). Les élevages caprins, ovins laitiers, porcins et de volailles sont plus représentés dans ce groupe ce qui explique le haut niveau du TA. Ces systèmes se caractérisent par une efficience faible du TA sur les herbivores ou du TS sur la surface fourragère. Malgré la part importante du bénévolat dans la réalisation du TA et du TS, la marge de manœuvre en temps est très faible $(690$ heures par an et pCB). Dans 20\% des cas, sans l'intervention de la maind'œuvre bénévole, la marge de manœuvre en temps serait nulle.

c) le lien entre logiques d'organisation, filières et composition de la $C B$

Les logiques d'organisation ne sont pas indépendantes des filières, ni de la composition de la cellule de base (figures 3 et 4) alors que ces deux variables ne font pas partie de l'analyse. Ces liens résultent des éléments présentés dans la première partie de nos résultats. $\mathrm{La}$ répartition des logiques d'organisation n'est pas homogène entre les régions (test du chi2 avec $\mathrm{P}<0,01)$. On observe ainsi la logique «partage» des grandes structures familiales dans les régions Champagne-Ardenne et Limousin, la logique «délégation» des grandes structures gérées par des éleveurs seuls en Bourgogne et en Lorraine, la logique «ajustement» des structures moyennes gérées par des couples en Basse Normandie et en Bretagne et celle «faible marge de manœuvre» des petites structures gérées par des éleveurs seuls en régions PACA et Aquitaine. En résumé, ce qui différencie les logiques d'organisation relève i) du dimensionnement de l'exploitation en lien avec la présence d'un atelier de grandes cultures, ii) de l'importance du travail d'astreinte en lien avec le système de production animale, iii) de l'adéquation entre la taille de l'exploitation et la taille de la cellule de base et $i v$ ) de la délégation du travail, donc du recours au salariat et au bénévolat, tout ceci conduisant à des marges de manœuvre en temps contrastées. 
Figure 4. Répartition des filières (en \%) selon les logiques d'organisation du travail.

$\begin{array}{llll}\square \text { Bovin viande } & \square \text { Ovin viande } & \square \text { Bovin lait } & \square \text { Ovin lait } \\ \square \text { Caprin lait } & \square \text { Caprin fromager } & \square \text { Porc } & \square \text { Volaille }\end{array}$

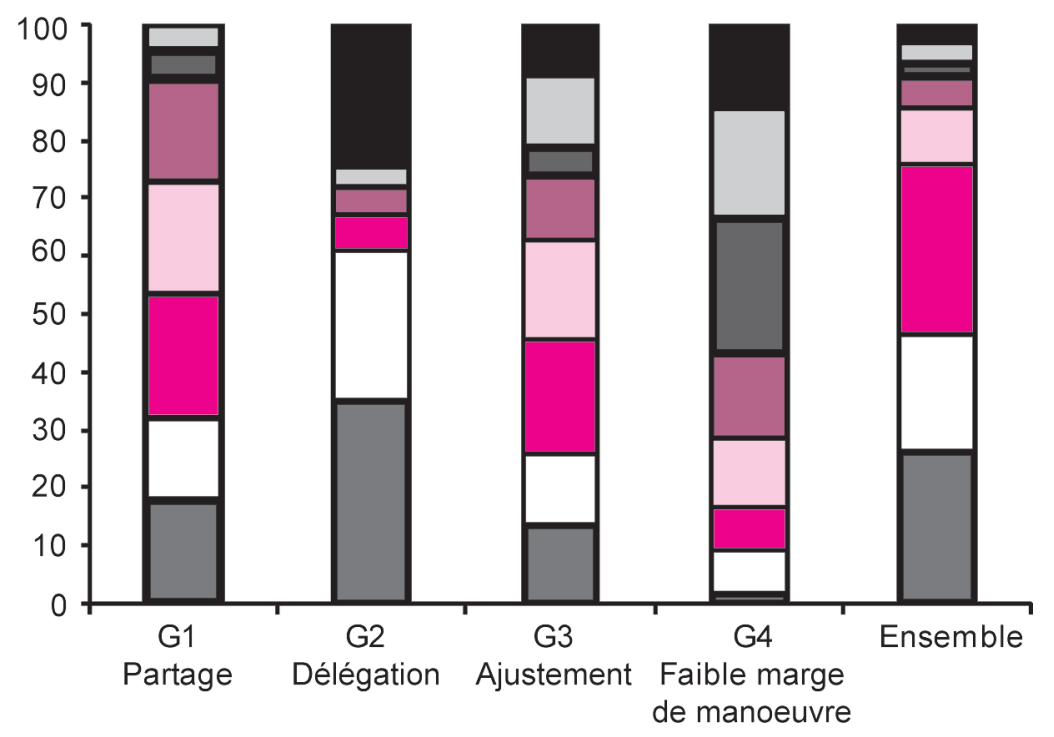

\section{3 / Limites et perspectives dégagées par l'analyse des Bilans Travail}

\section{1 / Malgré ses limites, l'analyse multifilière enrichit l'approche du travail en exploitations}

En nous appuyant sur le dispositif des Réseaux d'élevage (Institut de l'Elevage Chambres d'Agriculture), nous avons privilégié la prise en compte d'une grande diversité de situations. Mais le déséquilibre des effectifs entre les huit filières de production n'a pas facilité l'analyse en donnant trop de poids aux filières bovines laitière et allaitante, et à la filière ovine allaitante. L'entrée par filière a des limites car les différences peuvent être fortes entre systèmes de production au sein d'une même filière, par exemple, entre élevages ovins laitiers des Pyrénées-Atlantiques et de l'Aveyron ou entre élevages bovins à viande naisseurs et naisseurs-engraisseurs. L'analyse des temps de travaux entre filières pose le problème de l'unité à utiliser pour rendre compte de la taille $\mathrm{du}$ troupeau et évaluer l'influence du dimensionnement ou encore l'efficience du travail relatif au troupeau. Le recours aux UGB, possible pour les élevages de ruminants, n'est pas complètement satisfaisant car on sait bien qu'une UGB en bovin allaitant n'a pas la même signification en terme de travail qu'une UGB caprine par exemple. Il est hors de propos en élevage monogastrique.

Notre analyse ne prend pas en compte la répartition du travail dans l'année. Or, un même niveau de travail d'astreinte global annuel peut être obtenu avec des répartitions différentes de ce travail au cours de la campagne. S'intéresser à ces répartitions sur l'année (Cournut et Jordan 2008) aurait enrichi l'analyse. Cela constitue une des pistes de poursuite de ce travail.

L'application de la méthode Bilan Travail aux filières monogastriques a permis de comprendre certaines de leurs spécificités comme l'importance du salariat et de la gestion des bandes d'animaux (Hostiou et al 2007, Grannec 2010, Gallot 2011). Elle a nécessité des adaptations pour évaluer le travail d'astreinte qui, dans les élevages de ruminants se fait après avoir découpé la campagne en périodes où le TA est identique, ce qui renvoie la plupart du temps à un découpage saisonnier de la campagne (hiver en bâtiment, période de pâturage par exemple), alors qu'en élevage monogastrique, le TA est rythmé par l'enchaînement des bandes d'animaux, ce qui nécessite de l'évaluer en prenant en compte le rythme non quotidien de certaines tâches (saillie toutes les 3 semaines ou départs des porcelets tous les 15 jours par exemple). Elle a aussi montré en quoi ces filières pouvaient rejoindre les autres en termes d'organisation du travail et tirer bénéfice de la confrontation des résultats. Il est par exemple étonnant de voir la proximité entre les filières bovines à viande et volailles sur la quantité de TA et la configuration de la $\mathrm{CB}$. Ces deux filières diffèrent par contre grandement sur les choix de répartition du travail entre $\mathrm{CB}$ et hors $\mathrm{CB}$.

\section{2 / Les filières renvoient à des modèles techniques et sociocul- turels différents}

L'analyse de l'organisation du travail dans notre échantillon a mis en évidence des spécificités liées aux filières qui renvoient au contenu technique du travail lequel conditionne les temps de travaux (Dedieu et Servière 2001). Par exemple, les élevages caprins fromagers cumulent traite, soins aux animaux, fabrication fromagère et commercialisation (Calland et Madelrieux 2010, Guinamard 2010) et doivent ainsi faire face à de lourdes charges de travail d'astreinte. A contrario, les élevages bovins allaitants, de par l'absence de la traite et une conduite par lots de leurs animaux, ont un travail d'astreinte beaucoup moins important (Pichereau et al 2004, Kentzel 2010). Mais d'autres dimensions sont en jeu car la façon d'organiser la répartition des tâches à réaliser au sein du collectif diffère fortement selon les filières. En témoignent l'autonomie relative des exploitations bovines laitières, l'importance du bénévolat dans les exploitations ovines laitières des Pyrénées (Morin 2010), ou encore la prédominance des cellules de base à deux personnes ou plus à la tête des exploitations laitières, liée à l'astreinte de la traite (Fagon et Sabatté 2011). La dimension filière recouvre des modèles à la fois techniques et socioculturels différents qui influencent les logiques d'organisation du travail. Ainsi, l'importance du bénévolat dans les exploitations ovines laitières des Pyrénées s'explique par la logique de transmission patrimoniale des exploitations dans cette région qui induit une forte participation des parents. A contrario, la forte autonomie de la cellule de base concernant les travaux liés aux animaux en filière bovine laitière renvoie au modèle $\mathrm{du}$ «bon éleveur laitier» pour qui la traite est au cœur du métier et ne peut être déléguée. De plus, ces filières sont associées à des bassins de production ancrés dans des territoires ayant leurs propres caractéristiques socio-économiques, pouvant jouer sur des choix d'organisation comme par exemple la délégation (Errington et Gasson 1996).

\section{3 / L'organisation du travail s'évalue en référence à la cellule de base}

Le nombre de $\mathrm{pCB}$ est le facteur principal de variation des critères d'organisation du travail (Cournut et Chauvat 2010a). Les lignes de force sont confirmées: plus on est nombreux, plus la surface et la dimension des unités de production est réduite par travailleur, moins on délègue, moins on a de travail à charge par personne, et plus le temps 
disponible est important. Certains s'en sortent bien en étant seuls, d'autres ne présentent pas des marges de manœuvre très importantes malgré un collectif important. L'analyse multivariée a mis en évidence des logiques d'organisation spécifiques aux éleveurs seuls, aux couples ou aux GAEC familiaux, ce qui confirme que le travail doit être analysé et évalué en référence au collectif qui gère l'exploitation (Dufour et al 2007, Béguin 2008, Olaizola et al 2008, Cournut et Hostiou 2010). La compréhension des cohérences propres à chacune des logiques d'organisation du travail indique qu'elles répondent à des objectifs différents et renvoient à des attentes des éleveurs également différentes en termes de travail (Cournut et Dedieu 2005, Sidot et al 2005, Seegers et al 2006). Ainsi dans la logique «partage», l'organisation, basée sur une répartition du travail entre les différents membres du collectif familial, ne donne pas forcément la priorité à l'efficience ou à la libération de temps libre, mais permet de travailler ensemble et facilite le cas échéant l'apprentissage des plus jeunes (Rault 2005). D'autres dimensions interviennent dans les choix d'organisation des éleveurs, comme leurs conceptions du travail (Pichereau et al 2004, Commandeur 2005, Dufour et al 2010), la volonté de travailler en famille ou en couple (Fiorelli et al 2007), de transmettre des savoirs (Rault 2005) ou le goût du travail avec les animaux (Porcher 2003).

\section{4 / Comprendre la construc- tion de la marge de manœuvre en temps}

Cette étude met en lumière la diversité des logiques d'organisation du travail et les marges de manœuvre associées. Si le travail d'astreinte est un facteur important dans la construction du TDC (Cournut et Chauvat 2010a), la diversité des logiques d'organisation identifiées montre que plusieurs voies sont possibles pour réduire le travail d'astreinte et se ménager du temps disponible. Cela peut être la recherche d'une meilleure adéquation entre les dimensions de l'exploitation et la main-d'œuvre disponible (logique «ajustement»), le choix d'un système peu gourmand en TA ou encore la délégation d'une partie du TA à des travailleurs hors $\mathrm{CB}$ (logique «délégation»).

Dans certains cas, la logique d'organisation conduit à des résultats médiocres voire préoccupants en termes de marge de manœuvre en temps. Différentes études ont montré de façon empirique que le seuil de vivabilité pour une exploitation d'élevage française s'établissait entre 900 et 1000 heures de TDC par an et par travailleur (Pichereau et al 2004, Murat
2009). En deçà de 900 heures on considère la situation comme plus difficilement vivable. L'importance du nombre d'exploitations ayant des niveaux de $\mathrm{TDC} / \mathrm{pCB}$ faibles $(30 \%$ ont des valeurs inférieures à $800 \mathrm{~h}$ ) dans notre échantillon interroge la vivabilité des exploitations car celles-ci appartiennent pour la grande majorité aux Réseaux d'élevage, dispositif ayant pour vocation la construction de références et portant sur des systèmes sélectionnés sur leur viabilité économique.

La quantité de travail fournie par les éleveurs apparaît de moins en moins acceptable à leurs yeux surtout quand ils se comparent aux autres catégories socioprofessionnelles (Dufour et Dedieu 2010). Des marges de progrès existent cependant. La prise en compte du travail devient ainsi primordiale dans l'accompagnement des transformations des élevages. Cela suggère aussi que la perception du travail et de sa quantification n'a pas la même signification selon les éleveurs et n'est pas forcément en lien avec l'évaluation qu'en font les chercheurs ou agents de développement (Colombani et al 2009).

\section{5 / Conséquences pour le conseil}

En mettant en évidence la diversité des logiques d'organisation, cette étude donne des pistes pour améliorer les pratiques d'accompagnement des agriculteurs dans l'organisation du travail. Ces résultats donnent des clés pour mieux appréhender la situation de chaque exploitation, améliorer l'évaluation des marges de progrès et la pertinence des solutions envisagées. Par exemple, la proposition de groupements d'employeurs apparaît pertinente pour des éleveurs ayant une logique de délégation. Elle l'est beaucoup moins pour ceux ayant une logique d'autonomie. Pour ces derniers, on s'attachera, lors de l'installation d'un enfant, à repenser les équilibres du système de manière à ce que celui-ci trouve sa place. On peut être plus attentif à la répartition des tâches dans des exploitations ayant une logique de partage. Quant aux éleveurs ayant une logique «faible marge de manœuvre», un examen approfondi de la situation semble requis.

L'identification de logiques ne rentrant pas dans les «canons» de la performance travail (c'est-à-dire supérieur à $900 \mathrm{~h}$ par an et par pCB, cf. ci-dessus) interroge les pratiques d'analyse des résultats de temps de travaux pour la construction des référentiels. Le plus souvent, c'est bien des pratiques relatives aux élevages les plus performants qui sont étudiées et mises en avant. Or, notre étude montre que la diversité des situations ne relève pas uniquement d'une plus ou moins bonne maîtrise du temps de travail ou de son temps libre. D'autres éléments comme le modèle local d'organisation dominant (Lemery 2003), l'environnement social (Cournut et al 2008), la famille (Rault 2005), ou encore la conception du travail des éleveurs (Dufour et Dedieu 2010) peuvent influencer le poids donné à cette quantification et à l'évaluation du temps libre.

\section{Conclusion}

La mise en perspective des données d'organisation du travail de huit filières de production animale a permis de révéler les spécificités de chacune d'elles; lesquelles renvoient à des modèles techniques mais aussi socioculturels différents. Des proximités inattendues dans les registres d'organisation entre exploitations des filières porcines et caprines fromagères, toutes deux chargées en travail d'astreinte et mobilisant de façon significative de la main-d'œuvre salariée, ont été mises en évidence. Une autre proximité concerne les élevages bovins allaitants et de volailles qui se distinguent de même sur leur faible charge de travail d'astreinte.

L'évaluation de la marge de manœuvre en temps a montré que les situations les plus problématiques se rencontrent dans les exploitations gérées par une seule personne et fortement chargées en TA, pour lesquelles l'aide d'un parent bénévole ne permet pas l'obtention d'une marge de manœuvre suffisante, et pose question sur la pérennité de l'exploitation.

Notre étude confirme l'intérêt d'une approche multicritère pour analyser l'organisation du travail. Elle a mis en lumière la diversité des logiques d'organisation du travail et leur lien avec la nature du collectif qui gère l'exploitation : il existe bien des logiques d'organisation propres aux collectifs familiaux, aux couples ou aux éleveurs seuls. Les quatre logiques d'organisation identifiées et associées à des marges de manœuvre en temps spécifiques, correspondent à des équilibres particuliers entre le choix d'une production, son dimensionnement, la combinaison éventuelle avec d'autres ateliers, la configuration du collectif de travail et la façon dont il est mobilisé dans les différents travaux.

Plusieurs pistes d'approfondissement ont été identifiées. Elles concernent l'explicitation et la validation des logiques d'organisation, en prenant en compte d'autres volets de l'organisation du travail et d'autres dimensions 
du travail, mais aussi en clarifiant le sens donné par les éleveurs à ces organisations et les attentes en terme de travail auxquelles elle sont sensées répondre. La mobilisation de ces logiques dans le cadre du conseil doit conduire à mieux raisonner les critères d'évaluation de l'organisation du travail pour permettre d'inscrire ces logiques dans la construction de références adaptées.

\section{Remerciements}

Cette étude a été réalisée dans le cadre du Réseau Mixte Technologique
«Travail en élevage», financé par le Ministère de l'Agriculture.

Nous remercions les éleveurs et les conseillers pour la réalisation des enquêtes, et Gérard Servière (Institut de l'Elevage) pour sa relecture.

\section{Références}

Agreste, 2011. Recensement Agricole 2010. Premières tendances. Agreste Primeur $\mathrm{n}^{\circ} 266$ http://agreste.agriculture.gouv.fr/IMG/pdf primeur266-2.pdf

Béguin E., 2008. Composition et organisation des collectifs de travail des exploitations laitières de Picardie. Institut de l'Elevage, Collection Résultats. 32p.

Calland B., Madelrieux S., 2010. Le travail dans les exploitations d'élevage fermier. Cas d'exploitations caprines des Alpes du Nord. Cah. Agric., 19, 331-337.

Caramelle-Holtz E., Chauvat S., Ethève F., Kentzel M., Moreau J.C., Morin E., 2004. Le travail dans les exploitations d'élevage d'Aquitaine. Technipel $\mathrm{N}^{\circ}$ 2004.57.027. 69p.

Colombani-Lachapelle G., Levallois R., Perrier J.P., 2009. L'organisation du travail sur les fermes laitières : un moyen d'améliorer la rentabilité et la qualité de vie ?, ACFAS, Ottawa http://www.traget.ulaval.ca/uploads/tx centrerecherche/confGCL1109 02.pdf

Commandeur M., 2005. Styles of pig farming and family labour in the Netherlands. J. Comp. Fam. Stud., 36, 391-398.

Correa P., Dieguez F., Dedieu B., Arbeletche P., Bartaburu D., Morales H., Tourrand J.F., 2011. Comprendre l'organisation du travail pour préciser les stratégies d'éleveurs laitiers uruguayens. In : Le travail en agriculture dans les sciences pour l'action. Béguin P., Dedieu B., Sabourin E. (Eds), L'Harmattan Editions, Paris, France, 135-153.

Cournut S., Chauvat S., 2010a. Work organisation in livestock farms and farm liveability, In: Building sustainable rural futures, the added value of systems approaches in times of change and uncertainty. Darnhofer I., Grötzer M. (Eds), $9^{\text {th }}$ Europ. IFSA Symp., Vienna, Austria, 1148-1157.

Cournut S., Chauvat S., 2010b. Qualifier la vivabilité des exploitations d'élevage de ruminants d'un point de vue du travail. Renc. Rech. Rum., 17, 425-428.

Cournut S., Dedieu, B., 2005. Simplification des conduites d'élevage en bovins laitiers. Cah. Agric., 14, 541-547.

Cournut S., Hostiou N., 2010. Adaptations des systèmes bovins laitiers pour réduire la contrainte travail. Une étude en Ségala. Cah. Agric., 19, 348-353.

Cournut S., Jordan A., 2008. Analyse de groupe des Bilans Travail, guide méthodologique. Institut de l'Elevage. 63p.

Cournut S., Hostiou N, Pailleux J.Y., Léger L., 2008. Quelles adaptations des systèmes laitiers pour réduire la contrainte «travail»? Renc. Rech. Rum., 15, 163-166.
Cournut S., Serviere G., Hostiou N., Chauvat S., Dedieu B., 2010. L'organisation du travail en exploitations familiales d'élevage. Enseignements d'une analyse comparée conduite en France, en Amérique latine et au Vietnam. Cah. Agric., 19, 338-347.

Dedieu B., Servière G., 2001. Organisation du travail et fonctionnement des systèmes d'élevage. Renc. Rech. Rum., 8, 245-250.

Dedieu B., Servière G., Chauvat S., Tchakerian E., 2000. Bilan Travail pour l'étude du fonctionnement des exploitations d'élevage. Méthode. Collection Lignes, Institut de l'Elevage, 27p.

Dedieu B., Servière G., Madelrieux S., Dobremez L., Cournut S., 2006. Comment appréhender conjointement les changements techniques et les changements du travail en élevage ? Cah. Agric., 15, 506-513.

Delaveau A., Perrot C., Béguin E., Léger F., 1999. Les Réseaux d'élevage pour le conseil et la prospective. In : Les systèmes de production ovine et caprine : organisation de l'élevage et rôle des structures de développement. Rubino R., Mohrand-Fehr P. (Eds). CIHEAM-IAMZ, Zaragoza, Espagne, 99-103.

Dieguez F., Morales H., Cournut S., 2010. La methode Bilan Travail pour 1'approche du fonctionnement des élevages extensifs uruguayens. Cah. Agric., 19, 316-322.

Dufour A., Dedieu B., 2010. Rapport au temps de travail et modes d'organisation en élevage laitier. Cah. Agric., 19, 377-382.

Dufour A., Hostiou N., Cournut S., Dedieu B., 2007. Le travail en élevage laitier : des conceptions, des noyaux organisateurs et des profils d'organisation variés. Renc. Rech. Rum., 14, 385-388.

Errington A, Gasson R., 1996. The increasing flexibility of the farm and horticultural workforce in England and Wales. J. Rural Stud., 12, 127-141.

Escofier B., Pagès J., 1998. Analyses factorielles simples et multiples. Dunod Editions, Paris, France, 284p.

Fagon J., Sabatté N., 2011. Référentiel travail en élevages bovins lait. Synthèse de 190 Bilans Travail. Collection RMT travail, Institut de l'Elevage. Référence 001150 018, 32p.

Ferris C.P., Frost J., Binnie R.C., Patterson D.C., 2006. Dairy cows performance and labour inputs associated with two silage feeding systems. Grass Forage Sci., 61, 304314.

Fiorelli C., Dedieu B., Pailleux J.Y., 2007. Explaining diversity of livestock farming management strategies of multiple-job-holders: importance of level of production objectives and role of farming in the household. Animal, 8, 1209-1218.

Gallot S., 2011. Référentiel travail en élevages volailles. Synthèse de 24 Bilans Travail réalisés en Bretagne et Pays de la Loire. Collection RMT travail, Institut de l'Elevage. Référence 001050 042, 16p.

Gleeson D., O'Brien B., O'Donovan K., 2008. The labour input associated with calf care on Irish dairy farms. Livest. Sci., 116, 82-89.

Grannec M.L., 2010. Référentiel travail en élevages porcins. Synthèse de 23 Bilans Travail réalisés en Bretagne et Pays de la Loire. Collection RMT travail, Institut de l'Elevage. Référence 001050 040, 20p.

Guinamard C., 2010. Référentiel travail en élevages caprins. Synthèse de 53 Bilans Travail en productions laitière et fromagère fermière. Institut de l'Élevage, Référence 001050024 , 20 p.

Hostiou N., Dedieu B., Pailleux J.Y., 2007. Le salariat en élevage porcin et les régulations du travail. Journ. Rech. Porcine, 39, 193-198.

Hostiou N., Pham K.D., Madelrieux S., Vu Trong B., Dedieu B. 2010. Relations entre organisation du travail et taille des exploitations laitières : une étude à Moc Chau (Vietnam). Cah. Agric., 19, 348-353.

Johnsen S., 2004. The redefinition of family farming: agricultural restructuring and farm adjustment in Waihemo, New Zealand. J. Rural stud., 20, 418-432.

Kentzel M., 2010. Référentiel travail en élevages bovins viande. Synthèse de 170 Bilans Travail. Collection RMT travail, Institut de l'Élevage. Référence 001050 027, 36p

Kling-Eveillard F., Serviere G., Sabatté N., Chauvat S., Dedieu B., 2010. The research and extension network work and livestock farming. In: Building sustainable rural futures, The added value of systems approaches in times of change and uncertainty. Darnhofer I., Grötzer M. (Eds), $9^{\text {th }}$ Eur. IFSA Symp., Vienna, Austria, 1158-1168.

Lemery B., 2003. Les agriculteurs dans la fabrique d'une nouvelle agriculture. Sociol. Trav., 45, 9-25.

Madelrieux S., Dedieu B., 2008. Qualification and assessment of work organization in livestock farms. Animal, 2, 453-446.

Morin E., 2010. Référentiel travail en élevages ovins lait. Synthèse de 62 Bilans Travail réalisés dans le bassin de Roquefort et dans les PyrénéesAtlantiques. Collection RMT travail, Institut de l'Élevage, Référence 001050 23. 20p.

Murat C., 2009. Le travail c'est ... ..., l'alléger, c'est la conserver. Référentiel quantitatif sur le travail. Synthèse de 130 Bilans travail en 
exploitations bovines, ovines, caprines en Aveyron. 38p.

Olaizola A.M., Chertouh T., Manrique E., 2008. Adoption of a new feeding technology in Mediterranean sheep farming systems: implications and economic evaluation. Small Rum. Res. 79, 137-145.

Pichereau F., Becherel F., Farrie J.P., Legendre J., Veron J., Lequenne D., Serviere G., Cournut S., Dedieu B., 2004. Fonctionnement des grands troupeaux de vaches allaitantes : analyse des déterminants structurels et techniques de l'organisation du travail. Renc. Rech. Rum., 11, 129-136.

Porcher J., 2003. Bien-être et souffrance en élevage : condition de vie au travail des personnes et des animaux. Sociol. Trav., 45, 27-43.
Rattin S., 2006. Le travail s'intensifie dans les exploitations professionnelles toujours agrandies. Agreste Cah., 4, 3-12.

Rault G., 2005. Approche sociologique du travail en élevage et questions sur le conseil agricole. In: Systèmes fourrages, systèmes d'élevage et Travail, AFPF (Eds), Paris, France, $29-36$

Seegers J., Chauvat S., N'Guyen The B. Clement B., 2003. Le travail d'astreinte en élevage bovin laitier. Synthèse nationale d'enquêtes bilan travail. Analyse des aspirations et des solutions envisagées par les éleveurs du dispositif Réseaux d'élevage. Réf RM 855, 56p.

Seegers J., Moreau J.C., Béguin E. Guillaumin A., Frappat B., 2006. Attentes des éleveurs laitiers vis-à-vis de leurs conditions de travail et évolution de leurs systèmes d'exploitation. Fourrages, 185, 3-16.

Sidot G., Moreau J.C., Guillaumin A., 2005. L'attente des éleveurs par rapport à l'évolution de leur métier et des conditions de travail. Fourrages, 181, 95-103.

SPAD: Software for Data Mining, Data Analysis and Text Mining. 7.0 Release. SPAD, Paris, France. www.spad.eu

Turlot A., Froidmont E, Cardoso C. Burny P. Bauraind C., Bouquiaux J.M., Ledur A., Mayeres P., Wyzen B., Wavreille J., 2011. Le travail : première approche dans des exploitations laitières wallonnes. Renc. Rech. Rum., $18,244$.

\section{Résumé}

S'intéresser au travail des éleveurs et à la vivabilité des exploitations devient primordial dans le contexte actuel d'interrogations fortes sur le renouvellement des éleveurs. Dans cette étude, nous qualifions l'organisation du travail de 630 exploitations réparties dans huit filières animales (bovin laitier et à viande, ovin laitier et à viande, caprin laitier et fromager, porc et volaille), spécialisées ou mixtes. Nous utilisons la méthode Bilan Travail qui quantifie par catégorie de main-d'œuvre, les temps de travaux relatifs à la conduite des troupeaux et des surfaces et évalue une marge de manœuvre en temps des exploitants. Chaque filière a ses spécificités renvoyant à des modèles techniques mais aussi socioculturels différents. Des proximités inattendues dans les leviers d'organisation mobilisés ont été mises en évidence entre filières herbivores et monogastriques. L'évaluation de la marge de manœuvre en temps montre que pour 30\% des exploitations enquêtées, la vivabilité est questionnée. La description de la diversité des logiques d'organisation montre qu'il y a plusieurs façons de se libérer de l'astreinte et de se ménager du temps libre. Les quatre logiques d'organisation identifiées, associées à des marges de manœuvre en temps différentes, correspondent à des équilibres particuliers entre choix d'une production, la combinaison éventuelle avec d'autres ateliers, la dimension de l'exploitation et la configuration du collectif de travail. Mobilisés dans l'accompagnement des éleveurs pour résoudre leurs problèmes de travail, ces résultats donnent des clés pour mieux appréhender la situation de chaque exploitation, améliorer l'évaluation des marges de progrès et la pertinence des solutions envisagées.

\section{Abstract}

The organisation of work in livestock farming: an analysis of 630 work assessments in eight production sectors

Qualifying the liveability of livestock farms from the work point of view is becoming an important issue in the present context of serious questioning regarding the replacement of livestock farmers. We analysed the diversity of work organisation on 630 French livestock farms from varied production sectors (herbivore and monogastric), being either specialized or mixed. We used the Work Assessment method, which quantifies the work duration and evaluates room for manoeuvre time for farmers. Every production sector has its specificities linked to different technical but also socio-cultural models. Some proximity has been founded between herbivore and monogastric sectors. The evaluation of the room for manoeuvre showed that for $30 \%$ of the farms surveyed, liveability is questioned. By describing the diversity of logic of work organisation, we showed that there are different ways to decrease the routine workload and get some available time. We identified four logics of work organisation leading to contrasted room for manoeuvre, which result from complex interactions between dimension and combination of activities, and collective workforce configuration. We discuss the contributions of such results for extension and development.

COURNUT S., CHAUVAT S., 2012. L'organisation du travail en exploitation d'élevage : analyse de 630 Bilans Travail réalisés dans huit filières animales. In : Numéro spécial, Travail en élevage. Hostiou N., Dedieu B., Baumont R. (Eds). INRA Prod. Anim., 25, 101-112. 